\title{
Functional genomic analysis of bile salt resistance in Enterococcus faecium
}

\author{
Xinglin Zhang ${ }^{*}$, Damien Bierschenk, Janetta Top, lacovos Anastasiou, Marc JM Bonten, Rob JL Willems \\ and Willem van Schaik
}

\begin{abstract}
Background: Enterococcus faecium is a Gram-positive commensal bacterium of the mammalian intestinal tract. In the last two decades it has also emerged as a multi-resistant nosocomial pathogen. In order to survive in and colonize the human intestinal tract $E$. faecium must resist the deleterious actions of bile. The molecular mechanisms exploited by this bacterium to tolerate bile are as yet unexplored.

Results: In this study we used a high-throughput quantitative screening approach of transposon mutant library, termed Microarray-based Transposon Mapping (M-TraM), to identify the genetic determinants required for resistance to bile salts in E. faecium E1162. The gene gltK, which is predicted to encode a glutamate/aspartate transport system permease protein, was identified by M-TraM to be involved in bile resistance. The role of GltK in bile salt resistance was confirmed by the subsequent observation that the deletion of gltK significantly sensitized $E$. faecium E1162 to bile salts. To further characterize the response of E. faecium E1162 to bile salts, we performed a transcriptome analysis to identify genes that are regulated by exposure to $0.02 \%$ bile salts. Exposure to bile salts resulted in major transcriptional rearrangements, predominantly in genes involved in carbohydrate, nucleotide and coenzyme transport and metabolism.
\end{abstract}

Conclusion: These findings add to a better understanding of the molecular mechanisms by which $E$. faecium responds and resists the antimicrobial action of bile salts.

Keywords: Enterococcus faecium, Bile resistance, Transposon mutant library, Transcriptome

\section{Background}

Enterococcus faecium is a common inhabitant of the intestines of humans and animals and is present in many different natural environments [1,2]. However, during the past two decades E. faecium has rapidly emerged as an important multi-drug resistant nosocomial pathogen around the world and is now frequently responsible for hospital-acquired bloodstream, urinary tract and surgical wound infections [3-5]. The establishment of high-level intestinal colonization by enterococci is a crucial step in a process that can finally lead towards nosocomial infections [5].

Enterococci are known as being highly tolerant to hostile environments including high temperature conditions and high salt concentrations [6]. Enterococci are

\footnotetext{
* Correspondence: x.zhang@umcutrecht.nl

Department of Medical Microbiology, University Medical Center Utrecht, Heidelberglaan 100; Room G04.527, Utrecht 3584 CX, The Netherlands
}

also relatively resistant to chemical disinfectants like chlorine, glutaraldehyde and alcohol [7-9]. In order to survive in and colonize the human intestinal tract, a bacterium must be able to adapt to the stressful conditions that occur in this environment. Bile represents a major challenge to the intestinal microflora. The human liver daily secretes up to one liter of bile which is stored in the gall bladder and exported into the intestine [10]. Bile is a complex mixture composed mainly of bile salts, phospholipids, cholesterol, proteins and bilirubin [11]. Bile salts are amphipathic molecules that act as detergents, aiding in lipid solubilization and digestion, but they also play a role in host defenses, as bile salts have potent antimicrobial properties that can cause damage to the DNA, proteins and membranes of enteric bacteria $[12,13]$. In both Gram-positive and Gram-negative bacteria the disruption of bile tolerance loci often leads to impaired intestinal survival [14-16], while a mutation resulting in high-level bile resistance of Escherichia

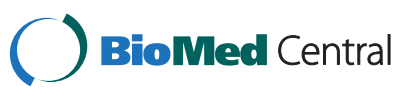


coli results in a fitness advantage during intestinal colonization [17].

As a successful colonizer of the intestinal tract, $E$. faecium must have developed mechanisms to sense, respond to and tolerate bile during its evolution as a gut commensal. Previously, two genetic loci $(\mathrm{gls} 33-\mathrm{glsB}$ and gls20-glsB1) that encode Gls-like proteins in E. faecalis and $E$. faecium were identified to be involved in bile resistance and pathogenicity in a mouse peritonitis model $[18,19]$. E. faecium was also possesses bile salt hydrolase activity [20], which is conferred by the protein encoded by the bsh gene (accession no. AY260046) [21]. In this study, we performed a genome-wide identification of the genetic loci required for bile salt resistance in E. faecium, using a high-throughput quantitative screening approach of transposon mutant libraries, termed Microarray-based Transposon Mapping (M-TraM) [22]. We also studied the transcriptional response of $E$. faecium to bile salts-induced stress.

\section{Methods}

Bacterial strains, plasmids and growth conditions

E. faecium and E. coli strains used in this study are listed in Table 1. The E. faecium strain E1162 was used throughout this study. This strain was isolated from a bloodstream infection in France in 1996 and its genome has previously been sequenced [23]. Unless otherwise mentioned, E. faecium was grown in brain heart infusion broth (BHI; Oxoid) at $37^{\circ} \mathrm{C}$. The E. coli strains $\mathrm{DH} 5 \alpha$ (Invitrogen) and EC1000 [24] were grown in LuriaBertani medium. Where necessary, antibiotics were used at the following concentrations: gentamicin at $300 \mu \mathrm{g}$ $\mathrm{ml}^{-1}$ for E. faecium and $25 \mu \mathrm{g} \mathrm{ml}^{-1}$ for E. coli, spectinomycin at $300 \mu \mathrm{g} \mathrm{ml}^{-1}$ for E. faecium and $100 \mu \mathrm{g} \mathrm{ml}^{-1}$ for E. coli. All antibiotics were obtained from Sigma-Aldrich (Saint Louis, MO). Growth of cultures was determined by measuring the optical density at $660 \mathrm{~nm}\left(\mathrm{OD}_{660}\right)$.

\section{Screening for genes involved in bile salt resistance using M-TraM}

M-TraM, a high throughput screening technique of transposon mutant libraries has previously been described in detail [22]. Here we use this technique to perform a genome-wide identification of genes involved in bile salt resistance in E. faecium. Briefly, aliquots containing approximately $10^{7}$ colony-forming units (CFU) from the mutant pool were used to inoculate $20 \mathrm{ml}$ of BHI broth or BHI broth supplemented with $0.02 \%$ bile salts (sodium cholate: sodium deoxycholate 1:1, Sigma-Aldrich). Cells were grown at $37^{\circ} \mathrm{C}$ for 20 hours, after which $1 \mathrm{ml}$ of the cultures were spun down and used for the extraction of genomic DNA, which was then further processed as described previously

Table 1 Strains and plasmids used in this study

\begin{tabular}{|c|c|c|}
\hline $\begin{array}{l}\text { Strain or } \\
\text { plasmid }\end{array}$ & Relevant characteristic(s) & $\begin{array}{l}\text { Source or } \\
\text { reference }\end{array}$ \\
\hline \multicolumn{3}{|l|}{ E. faecium } \\
\hline E1162 & Clinical isolate (bloodstream infection), isolated in France, 1996 & [23] \\
\hline$\Delta g / t K$ & Markerless deletion mutant of gltK gene of E1162 & This study \\
\hline$\triangle g s p A$ & Markerless deletion mutant of gspA gene of E1162 & This study \\
\hline$\Delta g l t K+g l t K$ & Complementation strain of $\Delta g / t K ; \Delta g l t K$ harboring pEF25-gltK & This study \\
\hline$\triangle g s p A+g s p A$ & Complementation strain of $\triangle g s p A ; \triangle g s p A$ harboring $p E F 25-g s p A$ & This study \\
\hline \multicolumn{3}{|l|}{ E. coli strains } \\
\hline $\mathrm{DH} 5 \mathrm{a}$ & E. coli host strain for routine cloning & Invitrogen \\
\hline EC1000 & MC1000 glgB::repA; host strain for pWS3 derived vectors & [24] \\
\hline \multicolumn{3}{|l|}{ Plasmids } \\
\hline pWS3 & Gram-positive thermosensitive origin of replication; $S p c^{r}$ & {$[29]$} \\
\hline pDEL3a & pWS3 carrying the $5^{\prime}$ and $3^{\prime}$ flanking regions of gene gltK for mutant construction & This study \\
\hline pDEL4a & pWS3 carrying the $5^{\prime}$ and $3^{\prime}$ flanking regions of gspA gene cluster for mutant construction & This study \\
\hline pDEL3b & $\begin{array}{l}\text { pDEL3a with a Gen }{ }^{r} \text { cassette which was flanked by lox66- and lox71-sites cloned between the } 5^{\prime} \text { and } 3^{\prime} \\
\text { flanking regions }\end{array}$ & This study \\
\hline PDEL4b & $\begin{array}{l}\text { pDEL4a with a Gen }{ }^{r} \text { cassette which was flanked by lox66- and lox71-sites cloned between the } 5^{\prime} \text { and } 3^{\prime} \\
\text { flanking regions }\end{array}$ & This study \\
\hline pWS3-Cre & pWS3 derivative expressing Cre in E. faecium & [22] \\
\hline pEF25 & Shuttle plasmid pAT18 with spectinomycin resistance cassette cloned in the EcoRI site; Spc ${ }^{r}$ Ery $^{r}$ & [30] \\
\hline pEF25-gltK & Complementation plasmid for $\Delta g / t K ; p E F 25$ carrying gltK & This study \\
\hline pEF25-gspA & Complementation plasmid for $\triangle g s p A ;$ pEF25 carrying gspA & This study \\
\hline
\end{tabular}


[22]. Statistical differences in hybridization signals between the conditions were analyzed using Cyber-T [25] (http:// cybert.microarray.ics.uci.edu/). Probes exhibiting a Bayesian P-value $<0.005$ were deemed statistically significant. A gene of which at least two identical probes (two different probes per gene were spotted in duplicate on the microarray [22]) passed this threshold were classified as significantly selected during exposure to bile salts. In an addition, genes which were selected between 0.5 - and 2-fold were deemed biologically insignificant and were filtered out. This experiment was performed with four biological replicates.

The microarray data generated in the M-TraM screening have been deposited in the ArrayExpress database (http://www.ebi.ac.uk/arrayexpress) under accession number E-MEXP-3797.

\section{Transcriptome profiling}

E. faecium E1162 was grown in $3 \mathrm{ml} \mathrm{BHI} \mathrm{broth} \mathrm{at} 37^{\circ} \mathrm{C}$ for 18 hours. Cultures were then diluted 100 fold in $20 \mathrm{ml}$ of prewarmed BHI broth (in a 50-ml Falcon tube) and grown until $\mathrm{OD}_{660}$ 0.3. Two ml aliquots of the cultures were centrifuged for 12 seconds at $16900 \mathrm{~g}$ at room temperature, and pellets were flash frozen in liquid $\mathrm{N}_{2}$ prior to RNA extraction. This sample served as the negative control ( $\mathrm{t}=0 \mathrm{~min}$ ) prior to the addition of bile salts. Bile salts (final concentration $0.02 \%$, w/v) were added into the remaining $18 \mathrm{ml}$ of culture. After 5 and 15 minutes of incubation at $37^{\circ} \mathrm{C}, 2 \mathrm{ml}$ aliquots of the cultures were centrifuged and flash frozen as described above. RNA isolation, cDNA synthesis and hybridization were performed as described in our previous work [22]. In this experiment, the expression of genes at $\mathrm{t}=5 \mathrm{~min}$ and $\mathrm{t}=15 \mathrm{~min}$ were compared to $\mathrm{t}=0 \mathrm{~min}$. Analysis for statistical significance was performed using the Webbased VAMPIRE microarray suite (http://sasquatch.ucsd. edu/vampire/) as described previously [26,27]. A gene of which all four probes (two different probes were spotted in duplicate on the microarray [22]) were identified as differentially expressed with a false discovery rate $<0.001$, were classified as significantly different between samples. Genes with an expression ratio between 0.5and 2-fold were deemed biologically insignificant and were filtered out. This experiment was performed with two biological replicates.

The microarray data generated in the transcriptome analysis have been deposited in the ArrayExpress database (http://www.ebi.ac.uk/arrayexpress) under accession number E-MEXP-3796.

\section{Construction of markerless deletion mutants and in trans complementation}

Markerless gene deletion mutants in the gltK gene (locustag: EfmE1162_1760) and the gspA gene (locustag: EfmE1162_1186) were created via the Cre-lox recombination system as previously described $[22,28]$. Briefly, the $5^{\prime}$ and 3' flanking regions (approximately $500 \mathrm{bp}$ each) of the target genes were PCR amplified with the primers in Table 2 . The two flanking regions were then fused together by fusion PCR (generating an EcoRI site between both fragments) and cloned into pWS3 [29], resulting in pDEL3a and pDEL4a (plasmids used or generated in this study are listed in Table 1). Then, a gentamicin-resistance cassette which was flanked by lox66- and lox71-sites [22] was cloned into the EcoRI site that was generated between the $5^{\prime}$ and $3^{\prime}$ flanking regions in pDEL3a and pDEL4a, respectively. The resulting plasmids pDEL3b and pDEL4b were then electrotransformed into E. faecium E1162. Marked deletion mutants were obtained by growing the gentamicinresistant transformants as described previously [22]. The plasmid pWS3-Cre [22], carrying a gene encoding Cre recombinase, was introduced into the marked mutant by electroporation. Further culturing for the removal of the gentamicin resistance cassette and subsequent loss of pWS-Cre was performed as described previously [22]. Excision of the gentamicin resistance cassette and loss of pWS3-Cre was verified by PCR using primers listed in Table 2.

In trans complemented strains of gltK and gspA gene deletion mutants were generated as described previously $[22,30]$. The gltK and gspA genes were PCR amplified, respectively, from the genomic DNA of E1162 using the primers listed in Table 2. The PCR products were cloned into the shuttle vector pEF25 [30]. The resulting plasmids, pEF25-gltK and pEF25-gspA, were introduced into the corresponding mutant strains by electroporation as described above.

\section{Determination of growth curves}

A BioScreen C instrument (Oy Growth Curves AB, Helsinki, Finland) was used to determine the effects of bile salts on bacterial growth. Wild type, mutants and the in trans complemented strains were grown overnight in BHI (containing appropriate antibiotics for the in trans complemented strains). Cells were inoculated at an initial $\mathrm{OD}_{660}$ of 0.0025 into $300 \mu \mathrm{BHI}$ and $\mathrm{BHI}$ with $0.02 \%, 0.04 \%, 0.08 \%$ and $0.16 \%$ of bile salts, respectively. The cultures were incubated in the Bioscreen $\mathrm{C}$ system at $37^{\circ} \mathrm{C}$ with continuous shaking, and absorbance of $600 \mathrm{~nm}\left(\mathrm{~A}_{600}\right)$ was recorded every $15 \mathrm{~min}$ for 12 hours. Each experiment was performed in triplicate.

\section{Bile salt adaptation and challenge assays}

To compare the sensitivity to bile salts of the parental strain E1162, the mutant strains and in trans complemented strains, overnight cultures were diluted 100 fold in fresh $\mathrm{BHI}$ and grown to $\mathrm{OD}_{660} 0.3$. One $\mathrm{ml}$ of the cell cultures were harvested by centrifugation at $12500 \mathrm{~g}$ for 1 minute and adapted to bile salts by resuspending the cells in BHI 
Table 2 Primers used in this study

\begin{tabular}{|c|c|}
\hline Primer & Sequence $^{\mathrm{a}}$ \\
\hline delete_Xmal_gltK_up_F & 5'-CCCCCCGGGCCAAGCAGGTACGATTGGAT-3' \\
\hline delete_EcoRI_gltK_up_R & 5'-AACCGGAAAGCAGAGAATTCTCGAAAACAATGAAACTTCAACA-3' \\
\hline delete_EcoRI_gltK_dn_F & 5'-TCGAGAATTCTCTGCTITCCGGTTACTTGG-3' \\
\hline delete_Xhol_gltK_dn_R & 5'-CCGCTCGAGGGAAGGATCACACCGATGAC-3' \\
\hline check_gltK_up & 5'-CGGAACGTTAATGGCAATCT-3' \\
\hline check_gltK_dn & 5'-CCGTACCAATCGTACCGATAA-3' \\
\hline delete_Xmal_gspA_up_F & 5'-CCCCCCGGGCCTCCTITTGGACTTTCTCG-3' \\
\hline delete_EcoRI_gspA_up_R & 5'-ACCACATTTAGCTGCAGAATTCGACGGCTTTCCGTTGTGTAG-3' \\
\hline delete_EcoRI_gspA_dn_F & 5'-CGAATTCTGCAGCTAAATGTGGTACGAA-3' \\
\hline delete_Xhol_gspA_dn_R & 5'-CCGCTCGAGGCCAAGTGAAAGCTTTGGAA-3' \\
\hline check_gspA_up & 5'-GCTCGAATTCTTCGATTGCT-3' \\
\hline check_gspA_dn & 5'-TGATGAGCCGTTAAATGGAA-3' \\
\hline complement_BamHI_gltK_F & 5'-ACGGGATCCTITTAGCAATCGTAGCTGGTTT-3' \\
\hline complement_Xhol_gltK_R & 5'-ACCGCTCGAGCGTGAATTTTCAAGTGCTC-3' \\
\hline complement_BamHI_gspA_F & 5'-ACGGGATCCTGAAAAACCTTCGATCGTTCA-3' \\
\hline complement_Xhol_gspA_R & 5'-ACCGCTCGAGTCCATTCCTACTCCCCCTCT-3' \\
\hline pAT392_EcoRI_lox66_genta_F & 5'-AGGGAATTCTACCGTTCGTATAGCATACATTATACGAAGTTATG ATAAACCCAGCGAACCATTTGAGG-3' \\
\hline pAT392_EcoRI_lox71_genta_R & 5'-CTCCGAATTCTACCGTTCGTATAATGTATGCTATACGAAGTTATT CAATCTITATAAGTCCTITAATAA-3' \\
\hline
\end{tabular}

${ }^{a}$ Restriction sites are underlined.

containing sub-lethal levels of bile salts $(0.02 \%)$ or in BHI without any additions. After a 15-minute adaptation period, viable counts were determined by serial dilution and plating on BHI agar plates (time point 0). Adapted and nonadapted cells were spun down as described above and resuspended in BHI containing 0.3\% bile salts, which corresponds to a concentration that is commonly reached in the human small intestine after ingestion of a meal [31]. After 5,30 and 60 minutes of incubation at $37^{\circ} \mathrm{C}$, aliquots of cells were washed with PBS and viable counts were determined following serial dilution and plating on BHI agar plates. The experiment was performed in triplicate and statistical analysis of the data was performed using an unpaired twotailed Student's $t$-test.

\section{Results and discussion}

Identification of genetic determinants involved in bile salt resistance in $E$. faecium by $\mathrm{M}$-TraM

To identify genes that are required for bile salt resistance in E. faecium E1162, we grew the pool of mutants in the presence or absence of a sub-lethal concentration $(0.02 \%)$ of bile salts for 20 hours, and used M-TraM to determine which mutants were less resistant to bile salts and therefore are selectively lost during culturing in the presence of bile salts. Seventy-five genes belonging to a variety of functional categories were identified to be involved in bile resistance (Additional file 1 and 2). A single gene, gltK (locus tag EfmE1162_1760), encoding a putative glutamate/aspartate transport system permease protein, was identified by M-TraM with the highest fold change (11.5 fold, which was notably higher than the other identified genes), indicating that this gene may contribute considerably to bile resistance in E. faecium. Consequently, we decided to further study the function of this gene in bile resistance (further described below). We were unable to find previous studies that linked GltK and its homologues in other microorganisms to bile resistance. BLAST analysis showed that GltK is conserved (with amino acid identities >97\%) in all of the 69 E. faecium genomes available (on 30 October 2012) at NCBI Genomes, indicating that the gltK gene is part of the E. faecium core genome. Another gene that was identified as contributing to bile resistance by M-TraM analysis was a gene (locus tag: EfmE1162_2043) encoding a putative cardiolipin synthetase, which functions as an enzyme in phospholipid metabolism and is involved in enterococcal daptomycin resistance [32,33]. It possibly acts by protecting the cells from membrane-associated damage induced by bile. In E. faecalis, the sagA gene was previously shown to be important in maintaining cell wall integrity and resistance to bile [34]. The E. faecium homolog (locus tag: EfmE1162_2437) of the sagA gene was also identified by M-TraM as potentially contributing to bile resistance. The bsh gene (locus tag: 
EfmE1162_2656) which encodes a bile salt hydrolase (BSH) [21] is conserved in all the 69 publicly available $E$. faecium genomes, including E1162. However this gene was not identified by M-TraM screening, presumably because BSH does not provide protection despite its predicted activity in the hydrolysis of bile salts. It is also possible that in the M-TraM screening, during which many different transposon insertion mutants are pooled together, the minor proportion of BSH-deficient mutants could be compensated by the extracellular bile salt hydrolase activity that is produced by cells that carry other mutations. We did not identify the two Gls-like protein-encoding loci which were shown to be involved in bile resistance in a previous study [19]. However, single deletions of either locus only resulted in a minor effect on bile salt resistance possibly due to mutual compensation of the two loci [19], which may also explain why we did not identify these loci in the M-TraM screening, as the mutant library only contains mutants that are inactivated in a single locus by transposon insertion [22].

\section{Transcriptional responses of $E$. faecium to bile salt- induced stress}

A microarray-based transcriptome analysis was used to identify genes that are regulated by exposure to bile salts. Compared to the untreated control, 214 (175 up-regulated and 39 down-regulated) and 190 (119 up-regulated and 71 down-regulated) genes were identified to be differentially expressed at $5 \mathrm{~min}$ and $15 \mathrm{~min}$ incubation with bile salts, respectively (Additional files 2 and 3). The data of the transcriptional analyses at the two different time points $(t=5 \mathrm{~min}$ and $t=15 \mathrm{~min})$ exhibited a correlation ( $\mathrm{R}^{2}$ of $\log _{2}$-transformed values) of 0.44 with each other (Additional file 4A). However the transcriptome data are completely uncorrlated with the M-TraM analysis $\left(R^{2}\right.$ of $\log _{2}$-transformed values $\left.\leq 0.001\right)$ (Additional file $4 \mathrm{BC}$ ), which is consistent with previous observations that gene expression poorly correlates with mutant fitness measurements $[22,35]$.

Genes identified at either time points were grouped by COG functional categories and the percentage abundance of each group was compared to the overall COGbased composition of the E1162 genome [23] (Figure 1 and Additional file 5). Genes in COG categories F (nucleotide transport and metabolism) and $\mathrm{H}$ (coenzyme transport and metabolism) were overrepresented among the down-regulated genes during exposure to bile salts, and no genes from these categories exhibited upregulated expression during bile salt exposure, indicating that the decreased expression of genes in these two functional categories is a major transcriptional response of E. faecium to bile salts. Among the up-regulated genes, genes in COG category $\mathrm{G}$ (carbohydrate transport and metabolism) were overrepresented at $5 \mathrm{~min}$ after exposure to bile salts. These included genes that are predicted to encode proteins involved in the utilization of a variety of sugars including maltose, maltodextrin, cellobiose, galactose, fructose, mannose and lactose. No genes from COG category $\mathrm{G}$ were down-regulated after 5 min. This observation indicates that bile salts positively impact on expression of genes involved in carbohydrate transport and metabolism immediately after exposure to bile salts, but this response becomes less prominent after longer periods of time. We also found a

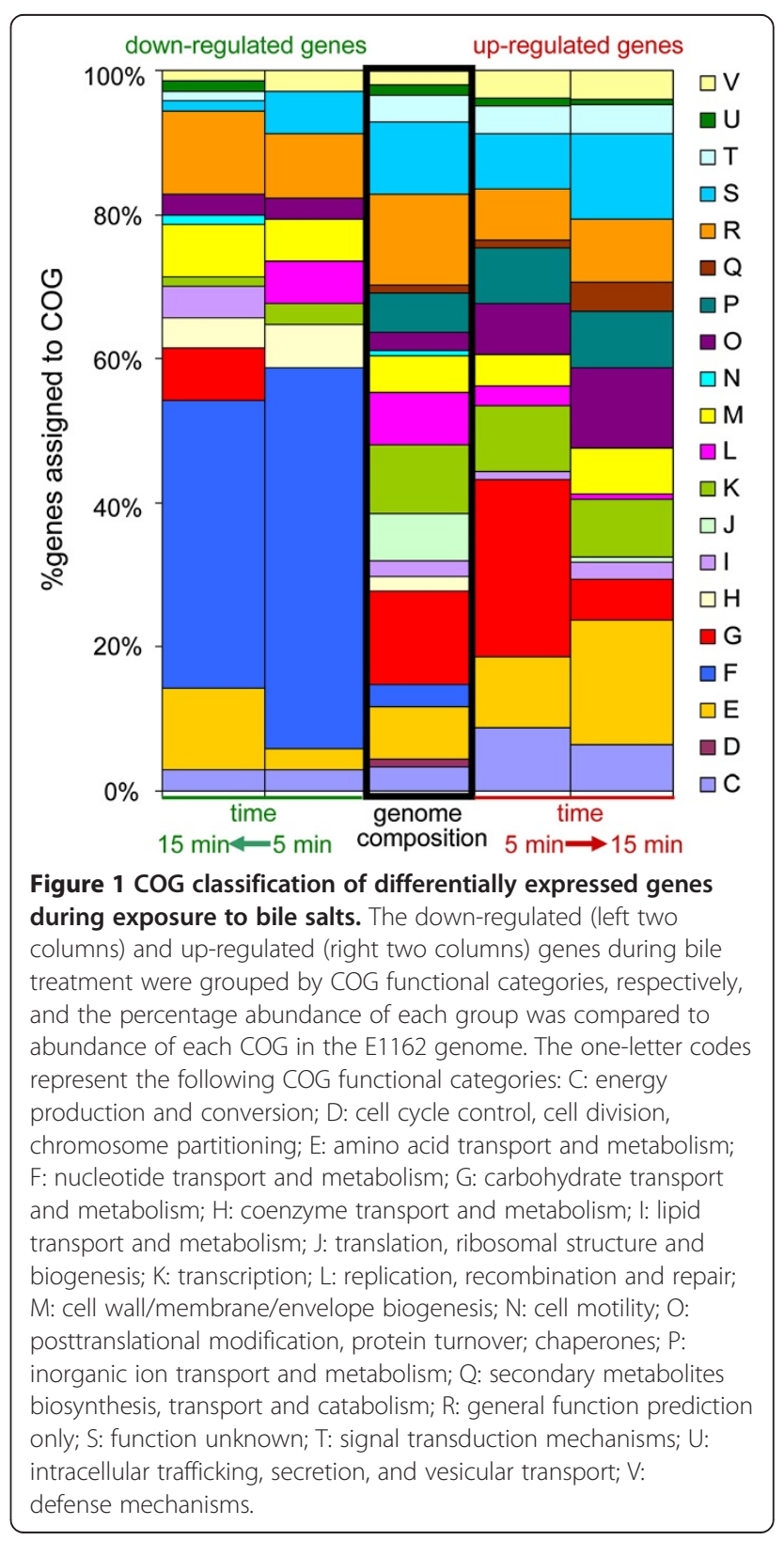


number of categories that were enriched in up-regulated genes, including $\mathrm{C}$ (energy production and conversion), $\mathrm{E}$ (amino acid transport and metabolism), $\mathrm{O}$ (posttranslational modification, protein turnover; chaperones) and Q (secondary metabolites biosynthesis, transport and catabolism). These data suggest an involvement of these functional categories in the E. faecium response to bile salts.

We further focused on a gene EfmE1162_1186 (gspA) which is predicted to encode a general stress protein A. This gene was identified by both transcriptome analysis (4.6 and 47.0 fold up-regulated at $5 \mathrm{~min}$ and $15 \mathrm{~min}$ of bile salts treatment, respectively) and M-TraM (2.8 fold less signal in bile-exposed library compared to the control). GspA is also highly conserved (with amino acid identities >98\%) in 66 of the 69 E. faecium genomes. We observed that both of the two Glslike protein-encoding loci (EfmE1162_1192-EfmE1162 _1193 and EfmE1162_1201-EfmE1162_1202) were induced over eight-fold during exposure to bile salts, although they were not identified by M-TraM screening. However, the $b s h$ gene was not identified to be differentially expressed in BHI with bile salts, indicating that the expression of this gene is not regulated by bile salts despite its predicted role in bile salt hydrolysis.

The transcriptional responses of E. faecalis to bovine bile has been investigated in a previous study [36]. A striking common finding of this study and our work is that a large gene cluster (locus tags EfmE1162_0724EfmE1162_0731 in E. faecium E1162 and EF1492EF1500 in E. faecalis V583), which putatively encodes a V-type ATPase, exhibits upregulated expression during exposure to bile salts. V-type ATPases are membrane proteins that function as proton- or sodium ion pumps that build up ion gradients at the expense of ATP [37]. Induction of this gene cluster suggested that E. faecium may generate a proton gradient to respond to bile medi- ated stress. The link between bile mediated stress and maintenance of the proton motive force (PMF) was previously demonstrated in the Gram-positive bacteria Lactobacillus plantarum [38], Bifidobacterium longum [39] and B. animalis [40]. Bile salts can induce DNA damage in bacteria, and consequently DNA mismatch repair proteins are important for bacterial bile resistance $[12,41,42]$. In this study we identified a gene (locus tag: EfmE1162_1335), encoding the DNA mismatch repair protein MutS, that was higher expressed (23.0 fold at 5 min and 9.5 fold at $15 \mathrm{~min}$ ) after addition of bile salts to the culture medium.

\section{Effect of bile salts on growth of E. faecium E1162 wild-type and $g l t K$ and $g s p A$ mutants}

To determine the role of GltK and GspA in bile salt resistance, markerless deletion mutants in gltK and gspA were constructed in E. faecium E1162, and the mutants were complemented in trans. The growth of E. faecium E1162 wild type (WT), the isogenic mutants and the complemented strains in BHI and BHI supplemented with bile salts were determined. In the absence of bile salts the wild-type strain and its isogenic mutants grew identically (Additional file 6). When these strains were grown in BHI with $0.02 \%, 0.04 \%, 0.08 \%, 0.16 \%$ and $0.32 \%$ bile salts, the growth rate of the $\Delta$ gltK mutant decreased compared to WT and this difference was most notable in BHI with $0.08 \%$ bile salts (Additional file 6 and Figure 2). The growth rate of the $\Delta g l t K$ mutant could be restored to WT levels upon in trans complementation (Figure 2), indicating that GltK contributes to bile resistance of E. faecium. Only very minor effects on growth rate and optical density in stationary phase were observed upon deletion of $g s p A$, indicating that this gene has an insignificant role in bile resistance of E. faecium despite its highly induced
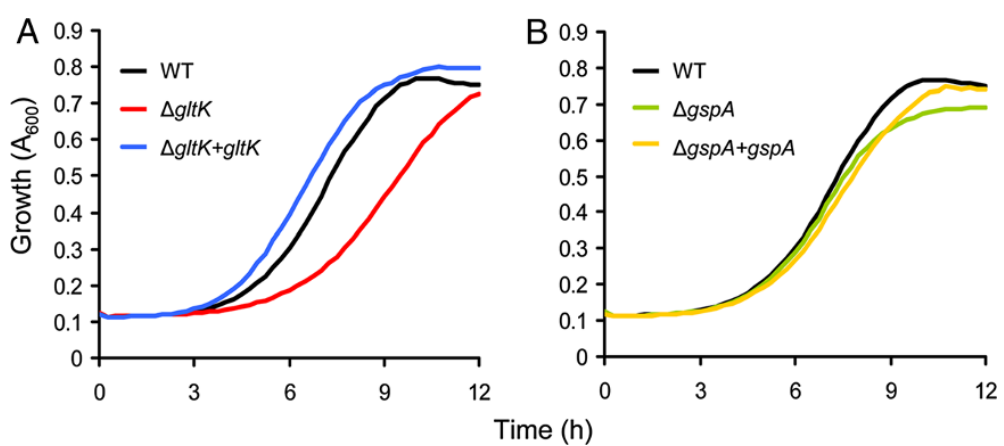

Figure 2 Growth of $\boldsymbol{E}$. faecium in $\mathbf{B H I}$ with $\mathbf{0 . 0 8} \%$ bile salts. Overnight cultures of E. faecium strains were inoculated at an initial cell density of $\mathrm{OD}_{660} 0.0025$ in BHI with $0.08 \%$ bile salts. Growth curves of wild-type E1162, the two mutants (panel A: $\triangle$ gltK, and panel B: $\triangle 9 s p A$ ) and in trans complemented strains are shown. Growth curves are the means of three independent experiments. 
expression upon exposure to bile salts. In addition, the sensitivity of both mutants to other stresses, including different pHs, oxidative and osmotic stress, were examined and no significant difference between the mutants and wild-type strain was detected (data not shown).

\section{Bile salt adaptation and challenge assays}

Exponential-phase cells of E1162 wild-type, the $\Delta g l t K$ mutant and the in trans complemented strain were adapted to $0.02 \%$ bile salts for $15 \mathrm{~min}$ or were left unadapted, and then challenged with $0.3 \%$ bile salts (Figure 3). Viable counts for the unadapted cells dropped below the detection limit $(<50 \mathrm{CFU} / \mathrm{ml})$ after the challenge, indicating that these cells were sensitive to this high concentration of bile salts. In contrast, the adapted cells were more tolerant to $0.3 \%$ bile salts, with $10^{4} \mathrm{CFU} / \mathrm{ml}$ surviving after $5 \mathrm{~min}$ of being exposed to bile salts and no significant further killing occurring during the remainder of the 1-hour experiment. These results showed that adaptation to low levels of bile salts provided E. faecium substantial protection to levels of bile salts that are lethal to non-adapted cells. The dele-

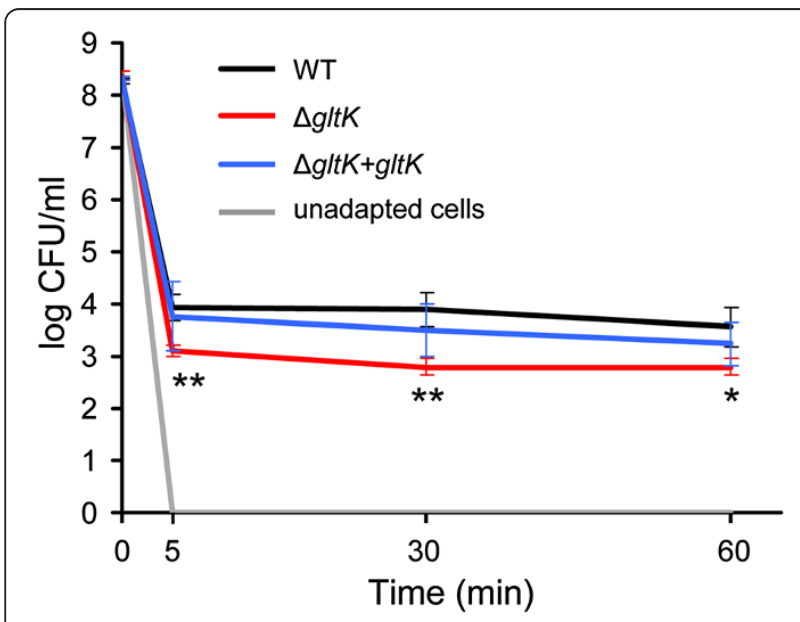

Figure 3 Survival of E. faecium cells following exposure to $0.3 \%$ bile salts with and without pre-adaptation. Exponential-phase cells of the E1162 wild type, the $\Delta g / t K$ mutant and its in trans complemented strain $\Delta g / t K+g / t K$ were adapted to $0.02 \%$ bile salts for 15 min or were left unadapted, and then challenged with $0.3 \%$ bile salts. Viable cells were determined immediately before exposure to $0.3 \%$ bile salts ( $t=0 \mathrm{~min}$ ) and after 5,30 and $60 \mathrm{~min}$ of challenge. The grey line represents unadapted cultures of these strains, in which viable counts dropped to undetectable levels ( $<50 \mathrm{CFU} / \mathrm{ml}$ ) after being challenged by bile salts. Bars represent the standard deviation of the mean of three independent experiments. Asterisks represent significant differences ( ${ }^{*}: P<0.05,{ }^{* *}: P<0.01$, as determined by an unpaired two-tailed Student's $t$-test) between the $\Delta g / t K$ mutant and the wild type strain. tion of $g l t K$ reduced the protection provided by the adaptation to a sub-lethal concentration of bile salts, leading to an approximately 1-log lower survival of pre-adapted gltK cells than the survival of the wild type E1162 cells. Survival of the in trans complemented strain upon pre-adaptation to bile salts was similar to that of the wild type. The $\Delta g s p A$ mutant was also included in this assay, but no significant difference was observed compared to wild-type E1162 (data not shown), again indicating that $g s p A$ was not required for bile resistance although its expression was highly induced by bile salts.

Our data suggest that bile salt-regulated genes do not necessarily contribute to bile resistance. Previous studies indicated that the protective adaptation to bile salts mainly results from changes in membrane composition and architecture that are independent of de novo protein synthesis $[43,44]$. Flahaut et al. showed that a 5 secondadaptation of E. faecalis to low level bile salts could provide substantial protection against challenge with lethal bile salt concentrations, and the addition of chloramphenicol during the adaptation period did not prevent development of acquired tolerance [44]. A similar result was also observed in L. monocytogenes [43]. However, the bile salt-regulated genes, rather than directly contributing to bile resistance, could be involved in other functions including virulence and carbohydrate metabolism [10]. It has previously been established in Salmonella [45,46] and Vibrio [47-49] that bile can be used as an environmental cue to influence the regulation genes involved in intestinal colonization and virulence. We identified many genes involved in carbohydrate metabolism that exhibited upregulated expression upon exposure to bile salts, e.g. a gene cluster (locus tags: EfmE1162_1484 EfmE1162_1489) putatively involved in maltose utilization (Zhang et al., unpublished work). This may suggest that E. faecium senses bile as an environmental signal indicating that it has entered the host gut, leading to a rapid adjustment of the cell's carbohydrate metabolism.

\section{Conclusions}

Responding and being resistant to bile are important features of bacteria that inhabit the gut [10]. In the present work, we have identified a genetic determinant in E. faecium that contributes to bile salt resistance, and studied the transcriptional response of E. faecium to bile salts. These findings add to a better understanding of the molecular mechanisms that lead to bile resistance in E. faecium.

\section{Additional files}

Additional file 1: Table S1. E. faecium genes involved in bile salt resistance as determined by M-TraM analysis. 
Additional file 2: Table S2. Complete data of gene expression ratios ( $\mathrm{t}=5 \mathrm{~min}$ and $\mathrm{t}=15 \mathrm{~min}$ versus $\mathrm{t}=0 \mathrm{~min}$ ) and $\mathrm{M}$-TraM analysis of $E$. faecium E1162.

Additional file 3: Table S3. Expression ratios $(t=5 \mathrm{~min}$ and $\mathrm{t}=15 \mathrm{~min}$ versus $t=0 \mathrm{~min}$ ) of $E$. faecium E1162 genes that are significantly differentially expressed upon exposure to bile salts.

Additional file 4: Figure S1. Comparison of transcriptome analysis (gene expression) and M-TraM analysis (mutant fitness). Each dot represents a gene probe. The axes represent the $\log _{2}$-transformed foldchanges in either transcriptome or M-TraM analysis. (A) Transcriptome ( $\mathrm{t}=15 \mathrm{~min}$ ) versus transcriptome $(\mathrm{t}=5 \mathrm{~min})$. (B) Transcriptome ( $\mathrm{t}=5 \mathrm{~min}$ ) versus $\mathrm{M}$-TraM. (C) Transcriptome $(\mathrm{t}=15 \mathrm{~min}$ ) versus M-TraM. (TIFF $376 \mathrm{~kb}$ )

Additional file 5: Table S4. COG classification of differentially expressed genes during exposure to bile salts.

Additional file 6: Figure S2. Growth of E. faecium in BHI with different concentrations of bile salts. Overnight cultures of $E$. faecium strains were inoculated at an initial cell density of $\mathrm{OD}_{660} 0.0025$ in $\mathrm{BHI}$ or $\mathrm{BHI}$ with $0.02 \%, 0.04 \%, 0.08 \%, 0.16 \%$ and $0.32 \%$ of bile salts. Growth curves of wild-type E1162 and the $\Delta g / t K$ mutant are shown.

\section{Competing interests}

The authors declare that they have no competing interests.

\section{Authors' contributions}

XZ and WVS designed research. XZ, DB, JT and IA performed experiments. $X Z, R J W$ and $W V S$ analyzed data. XZ, RJW, MJMB and WVS wrote the manuscript. All authors read and approved the final manuscript.

\section{Acknowledgments}

This work was supported by the Nederlandse Organisatie voor Wetenschappelijk Onderzoek [NWO-VENI grant 916.86.044] and the European Union Seventh Framework Programme (FP7-HEALTH-2011-single-stage) Evolution and Transfer of Antibiotic Resistance (EvoTAR) [grant agreement number 282004].

Received: 16 January 2013 Accepted: 18 April 2013

Published: 3 May 2013

\section{References}

1. Top J, Willems R, Bonten M: Emergence of CC17 Enterococcus faecium: from commensal to hospital-adapted pathogen. FEMS Immunol Med Microbiol 2008, 52(3):297-308.

2. Sghir A, Gramet G, Suau A, Rochet V, Pochart P, Dore J: Quantification of bacterial groups within human fecal flora by oligonucleotide probe hybridization. Appl Environ Microbiol 2000, 66(5):2263-2266

3. Willems RJ, Top J, Van Schaik W, Leavis H, Bonten M, Siren J, Hanage WP Corander J: Restricted gene flow among hospital subpopulations of Enterococcus faecium. MBio 2012, 3(4).

4. Willems RJ, van Schaik W: Transition of Enterococcus faecium from commensal organism to nosocomial pathogen. Future Microbiol 2009, 4(9):1125-1135.

5. Arias CA, Murray BE: The rise of the Enterococcus: beyond vancomycin resistance. Nat Rev Microbiol 2012, 10(4):266-278.

6. Facklam RR, Collins MD: Identification of Enterococcus species isolated from human infections by a conventional test scheme. J Clin Microbiol 1989, 27(4):731-734.

7. Bradley CR, Fraise AP: Heat and chemical resistance of enterococci. J Hosp Infect 1996, 34(3):191-196.

8. Freeman R, Kearns AM, Lightfoot NF: Heat resistance of nosocomial enterococci. Lancet 1994, 344(8914):64-65.

9. Kearns AM, Freeman R, Lightfoot NF: Nosocomial enterococci: resistance to heat and sodium hypochlorite. J Hosp Infect 1995, 30(3):193-199.

10. Begley M, Gahan CG, Hill C: The interaction between bacteria and bile. FEMS Microbiol Rev 2005, 29(4):625-651.

11. Esteller A: Physiology of bile secretion. World J Gastroenterol 2008, 14(37):5641-5649.

12. Merritt ME, Donaldson JR: Effect of bile salts on the DNA and membrane integrity of enteric bacteria. J Med Microbiol 2009, 58(Pt 12):1533-1541.
13. Hofmann AF, Hagey LR: Bile acids: chemistry, pathochemistry, biology, pathobiology, and therapeutics. Cell Mol Life Sci 2008, 65(16):2461-2483.

14. Begley M, Sleator RD, Gahan CG, Hill C: Contribution of three bileassociated loci, $b s h, p v a$, and $b t \mid B$, to gastrointestinal persistence and bile tolerance of Listeria monocytogenes. Infect Immun 2005, 73(2):894-904

15. Kristich CJ, Wells CL, Dunny GM: A eukaryotic-type Ser/Thr kinase in Enterococcus faecalis mediates antimicrobial resistance and intestinal persistence. Proc Natl Acad Sci USA 2007, 104(9):3508-3513.

16. Reynolds MM, Bogomolnaya L, Guo J, Aldrich L, Bokhari D, Santiviago CA, McClelland $M$, Andrews-Polymenis $\mathrm{H}$ : Abrogation of the twin arginine transport system in Salmonella enterica serovar Typhimurium leads to colonization defects during infection. PLoS One 2011, 6(1):e15800.

17. De Paepe M, Gaboriau-Routhiau V, Rainteau D, Rakotobe S, Taddei F, Cerf-Bensussan N: Trade-off between bile resistance and nutritional competence drives Escherichia coli diversification in the mouse gut. PLoS Genet 2011, 7(6):e1002107.

18. Teng F, Nannini EC, Murray BE: Importance of g/s24 in virulence and stress response of Enterococcus faecalis and use of the Gls24 protein as a possible immunotherapy target. J Infect Dis 2005, 191(3):472-480.

19. Choudhury T, Singh KV, Sillanpaa J, Nallapareddy SR, Murray BE: Importance of two Enterococcus faecium loci encoding Gls-like proteins for in vitro bile salts stress response and virulence. J Infect Dis 2011, 203(8):1147-1154.

20. Franz CM, Specht I, Haberer P, Holzapfel WH: Bile salt hydrolase activity of Enterococci isolated from food: screening and quantitative determination. J Food Prot 2001, 64(5):725-729.

21. Wijaya A, Hermann A, Abriouel H, Specht I, Yousif NM, Holzapfel WH, Franz CM: Cloning of the bile salt hydrolase (bsh) gene from Enterococcus faecium FAIR-E 345 and chromosomal location of bsh genes in food enterococci. J Food Prot 2004, 67(12):2772-2778.

22. Zhang X, Paganelli FL, Bierschenk D, Kuipers A, Bonten MJ, Willems RJ, van Schaik W: Genome-wide identification of ampicillin resistance determinants in Enterococcus faecium. PLoS Genet 2012, 8(6):e1002804.

23. van Schaik W, Top J, Riley DR, Boekhorst J, Vrijenhoek JE, Schapendonk CM, Hendrickx AP, Nijman IJ, Bonten MJ, Tettelin H, et al: Pyrosequencingbased comparative genome analysis of the nosocomial pathogen Enterococcus faecium and identification of a large transferable pathogenicity island. BMC Genomics 2010, 11:239.

24. Leenhouts K, Buist G, Bolhuis A, ten Berge A, Kiel J, Mierau I, Dabrowska M, Venema G, Kok J: A general system for generating unlabelled gene replacements in bacterial chromosomes. Mol Gen Genet 1996, 253(1-2):217-224.

25. Baldi P, Long AD: A Bayesian framework for the analysis of microarray expression data: regularized $t$-test and statistical inferences of gene changes. Bioinformatics 2001, 17(6):509-519.

26. Lebreton F, van Schaik W, Sanguinetti M, Posteraro B, Torelli R, Le Bras F, Verneuil N, Zhang X, Giard JC, Dhalluin A, et al: AsrR is an oxidative stress sensing regulator modulating Enterococcus faecium opportunistic traits, antimicrobial resistance, and pathogenicity. PLoS Pathog 2012 8(8):e1002834

27. Hsiao A, Ideker T, Olefsky JM, Subramaniam S: VAMPIRE microarray suite: a web-based platform for the interpretation of gene expression data. Nucleic Acids Res 2005, 33(Web Server issue):W627-632.

28. Sauer B: Functional expression of the cre-lox site-specific recombination system in the yeast Saccharomyces cerevisiae. Mol Cell Biol 1987, 7(6):2087-2096.

29. Zhang X, Vrijenhoek JE, Bonten MJ, Willems RJ, van Schaik W: A genetic element present on megaplasmids allows Enterococcus faecium to use raffinose as carbon source. Environ Microbiol 2011, 13(2):518-528

30. Top J, Sinnige JC, Majoor EA, Bonten MJ, Willems RJ, van Schaik W: The recombinase IntA is required for excision of esp-containing ICEEfm 1 in Enterococcus faecium. J Bacteriol 2011, 193(4):1003-1006.

31. Van Deest BW, Fordtran JS, Morawski SG, Wilson JD: Bile salt and micellar fat concentration in proximal small bowel contents of ileectomy patients. J Clin Invest 1968, 47(6):1314-1324.

32. Palmer KL, Daniel A, Hardy C, Silverman J, Gilmore MS: Genetic basis for daptomycin resistance in enterococci. Antimicrob Agents Chemother 2011 , 55(7):3345-3356.

33. Arias $C A$, Panesso D, McGrath DM, Qin X, Mojica MF, Miller C, Diaz L, Tran TT, Rincon S, Barbu EM, et al: Genetic basis for in vivo daptomycin resistance in enterococci. N Engl J Med 2011, 365(10):892-900. 
34. Breton $Y L$, Maze A, Hartke A, Lemarinier S, Auffray $Y$, Rince A: Isolation and characterization of bile salts-sensitive mutants of Enterococcus faecalis. Curr Microbiol 2002, 45(6):434-439.

35. Deutschbauer A, Price MN, Wetmore KM, Shao W, Baumohl JK, Xu Z, Nguyen M, Tamse R, Davis RW, Arkin AP: Evidence-based annotation of gene function in Shewanella oneidensis MR-1 using genome-wide fitness profiling across 121 conditions. PLoS Genet 2011, 7(11):e1002385.

36. Solheim M, Aakra A, Vebo H, Snipen L, Nes IF: Transcriptional responses of Enterococcus faecalis V583 to bovine bile and sodium dodecyl sulfate. Appl Environ Microbiol 2007, 73(18):5767-5774.

37. Senior AE: The proton-translocating ATPase of Escherichia coli. Annu Rev Biophys Biophys Chem 1990, 19:7-41.

38. Bron PA, Molenaar D, de Vos WM, Kleerebezem M: DNA micro-array-based identification of bile-responsive genes in Lactobacillus plantarum. J Appl Microbiol 2006, 100(4):728-738.

39. Sanchez B, Champomier-Verges MC, Anglade P, Baraige F, de Los ReyesGavilan CG, Margolles A, Zagorec M: Proteomic analysis of global changes in protein expression during bile salt exposure of Bifidobacterium longum NCIMB 8809. J Bacterio/ 2005, 187(16):5799-5808.

40. Sanchez B, Reyes-Gavilan CG DI, Margolles A: The F1F0-ATPase of Bifidobacterium animalis is involved in bile tolerance. Environ Microbiol 2006, 8(10):1825-1833.

41. Payne A, Schmidt TB, Nanduri B, Pendarvis K, Pittman JR, Thornton JA, Grissett J, Donaldson JR: Proteomic analysis of the response of Listeria monocytogenes to bile salts under anaerobic conditions. J Med Microbio/ 2013, 62(Pt 1):25-35.

42. Prieto Al, Ramos-Morales F, Casadesus J: Bile-induced DNA damage in Salmonella enterica. Genetics 2004, 168(4):1787-1794.

43. Begley M, Gahan CG, Hill C: Bile stress response in Listeria monocytogenes LO28: adaptation, cross-protection, and identification of genetic loci involved in bile resistance. Appl Environ Microbiol 2002, 68(12):6005-6012.

44. Flahaut S, Hartke A, Giard JC, Benachour A, Boutibonnes P, Auffray Y: Relationship between stress response toward bile salts, acid and heat treatment in Enterococcus faecalis. FEMS Microbiol Lett 1996, 138(1):49-54.

45. Prouty AM, Brodsky IE, Manos J, Belas R, Falkow S, Gunn JS: Transcriptional regulation of Salmonella enterica serovar Typhimurium genes by bile. FEMS Immunol Med Microbiol 2004, 41(2):177-185.

46. Prouty AM, Gunn JS: Salmonella enterica serovar typhimurium invasion is repressed in the presence of bile. Infect Immun 2000, 68(12):6763-6769.

47. Krukonis ES, DiRita VJ: From motility to virulence: Sensing and responding to environmental signals in Vibrio cholerae. Curr Opin Microbiol 2003, 6(2):186-190.

48. Schuhmacher DA, Klose KE: Environmental signals modulate ToxTdependent virulence factor expression in Vibrio cholerae. J Bacteriol 1999, 181(5):1508-1514.

49. Gupta $\mathrm{S}$, Chowdhury R: Bile affects production of virulence factors and motility of Vibrio cholerae. Infect Immun 1997, 65(3):1131-1134.

\section{Submit your next manuscript to BioMed Central and take full advantage of:}

- Convenient online submission

- Thorough peer review

- No space constraints or color figure charges

- Immediate publication on acceptance

- Inclusion in PubMed, CAS, Scopus and Google Scholar

- Research which is freely available for redistribution

Submit your manuscript at www.biomedcentral.com/submit
C BioMed Central 